\title{
Investigation of Mitomycin-C-treated Fibroblasts in 3-D Collagen Gel and Conditioned Medium for Keratinocyte Proliferation
}

\author{
*Yi-Chau Huang, *Tzu-Wei Wang, †\$Jui-Sheng Sun, and *§Feng-Huei Lin \\ *Institute of Biomedical Engineering, College of Medicine and College of Engineering, National Taiwan University; †Institute \\ of Rehabilitation Science and Technology, National Yang-Ming University; $\neq$ Department of Orthopedic Surgery, Taipei \\ Municipal Yang-Ming Hospital; and \$Department of Biomedical Engineering, National Taiwan University Hospital, Taipei,
}

Taiwan

\begin{abstract}
Fibroblasts produce a spectrum of necessary growth factors essential for growth and proliferation of a variety of cell types. In this study, the paracrine effect of mitomycin-C-treated fibroblasts with various densities in collagen gel for keratinocyte proliferation was investigated from which an optimum cell density and optimum conditioned medium would be determined to expand keratinocyte without further differentiation for skin equivalent tissue engineering. The optimum cell density in collagen feeder gel for optimum collected medium preparation will be determined by checking the level of keratinocyte growth factor and granulocyte macrophage colony-stimulating factor in conventional medium. The results showed that the cell density of $1 \times 10^{5}$ cells/gel in the feeder gel is better to produce optimum collected medium. The conditioned medium is prepared by mixing together the optimum collected medium and molecular cellular and developmental biology (MCDB) 153 medium in different ratios for keratinocyte growth. The keratinocyte viability will be measured by 3-(4,5-dimethyl-thiazol-2-yl)-2,5diphenyltetrazolium bromide (MTT) assay to determine the optimum conditioned medium. From the study, 67\% conditioned medium was supposed as the better medium for keratinocyte proliferation. In this experiment, the opti-
\end{abstract}

mum cell density in feeder gel to coculture with keratinocytes is also determined as $1 \times 10^{5}$ cells/gel. Keratin 10 (K10) and Terminal Deoxynucleotidyl Transferase Mediated dUTP Nick End Labeling stain will be used to check the cell differentiation and apoptosis, respectively. The results suggest that keratinocytes should not be cultured in postconfluent conditions due to undesired apoptosis and differentiation. The result of cell viability from passages to passages shows that the optimum feeder gel plays a more important role to the keratinocyte proliferation than that of optimum conditioned medium. Keratinocytes cultured with optimum feeder gel in $67 \%$ conditioned medium could effectively promote proliferation, inhibit apoptosis, and prevent differentiation. The combination of conditioned media and feeder gel to culture keratinocytes without external supplements can provide an inexpensive way for keratinocyte proliferation and construct an environment for real-time communication between the two cells. The results conclude that keratinocyte cultivation in feeder gel with modified medium should be feasible in the production of high quality keratinocytes for skin equivalents preparation. Key Words: Conditioned medium -Collagen gel-Keratinocyte-Proliferation-Cell-cell interactions.
There are over one million extensive burn patients in the U.S.A. each year, and 3900 of them died due to shortage of skin grafts (1). Several dressings such as autologous, allogenous, xenogenous, and artificial wound dressing have been developed as skin treatment for extensive burn patients. However, none of

Received September 2005; revised November 2005.

Address correspondence and reprint requests to Dr. Feng-Huei Lin, Institute of Biomedical Engineering, National Taiwan University, No. 1, Sec. 1, Jen-Ai Road, Taipei, Taiwan. E-mail: double@ ha.mc.ntu.edu.tw these dressings completely satisfied clinical demands. Autologous dressing is always in short supply due to limited area of normal skin from extensive burn patients. Although allogenous dressing has excellent healing rate, this type of dressing is very expensive and does not assist epidermis growth. Xenogenous dressing always had problems with immuno-rejection. Artificial wound dressing is expensive and requires additional epidermis grafted from a burn patient.

Currently available skin grafts all need a patient's own epidermis to help full recovery. Tissueengineered skin equivalent has been developed to 
achieve complete healing of burn patients. However, harvesting sufficient keratinocytes to cover dermal equivalent is a major obstacle in producing high quality skin equivalents. Keratinocytes must maintain their phenotype without any undesired differentiations throughout the steps of harvest, isolation, and proliferation.

There are many methods to overcome the abovementioned difficulties. Harvested keratinocytes from burn patients are subcultivated several times until sufficient in number to cover the surface of the dermal equivalent. However, adult primary keratinocytes have a lower proliferation rate for expansion economically. Keratinocytes tend to apoptosis or lose phenotype after many stages of expansion on monolayer culture (2).

Several studies have been investigated to add growth factor (e.g., epidermal growth factor [EGF]) into a monolayer culture system. Growth factors can increase proliferation but they can be expensive and short-lived. It is not feasible to provide exogenous growth factors to keratinocytes at optimum levels. Although this method has had limited success, it is not yet a reality (3-5).

Primary human dermal fibroblasts have been seeded as feeder layers (6), which produce a variety of necessary growth factors for keratinocyte proliferation (7-9). In a simple two-dimensional feeder layer coculture system, keratinocytes are seeded on the postmitotic dermal fibroblasts feeder layer for proliferation. Nonetheless, the culture system is unstable because of two distinct cell types during a long culturing period $(10,11)$.

The collagen gel has been mixed with dermal fibroblasts to achieve a functional three-dimensional (3-D) dermal equivalent. Keratinocytes are seeded subsequently to develop the full thickness skin equivalent. Viable fibroblasts in the collagen gel produce extracellular matrix components for matrix reorganization, and fibroblast secret paracrine mediators to regulate keratinocyte proliferation (12). From those studies, we understand that the paracrine mediators might enhance keratinocyte proliferation via cell signals between dermal fibroblasts and keratinocytes.

In this study, we will create a method to expand keratinocytes without lost phenotype or apoptosis. If dermal fibroblast density and activity are well controlled (13), we can study the effect of dermal fibroblast paracrine mediators on the keratinocyte proliferation. In this study, the mitomycin-C (MMC)treated fibroblasts are mixed with collagen gel as a feeder gel and cultured in trans-well for a period of time. The cultured medium is subsequently collected as conditioned medium. The keratinocytes are then cocultured with feeder gel with various concentrations of conditioned medium. These MMC-treated fibroblasts in 3-D collagen gel can continuously provide necessary growth factors to improve proliferation rates for keratinocytes.

Keratinocyte growth factor (KGF) and granulocyte macrophage-colony stimulating factor (GM-CSF) have also been investigated in fibroblastkeratinocyte communications. This study will aim to elucidate the possible functions of the two growth factors on the keratinocyte proliferation. The following will be addressed: (i) the optimum cell density in the collagen gel to produce appropriate conditioned medium (optimum feeder gel); (ii) the optimum concentration of conditioned medium for the keratinocyte growth (optimum conditioned medium); and (iii) the importance of optimum feeder and optimum conditioned medium.

The whole system and design are shown in Fig. 1. The system and design should provide an economic and fast method to effectively promote keratinocyte proliferation to produce enough number of keratinocytes for skin equivalent tissue engineering. In addition, the 3-D collagen gels with MMC-treated fibroblasts can continuously secrete stimulating factors for keratinocyte growth.

\section{MATERIALS AND METHODS}

\section{Keratinocyte isolation and culture}

Split thickness skin was obtained from patients undergoing breast reduction surgery or abdominoplasties. The skin was sectioned into $1 \mathrm{~cm}^{2}$ pieces and incubated overnight (18-24 h) at $4^{\circ} \mathrm{C}$ in MCDB 153 serum-free medium (Sigma, St. Louis, MO, U.S.A.) supplemented with $36 \mathrm{U}$ per $\mathrm{mL}$ thermolysin and $1 \%$ penicillin/streptomycin. The epidermal and dermal layers were carefully separated using forceps. A scalpel blade was utilized to gently scrape keratinocytes under the surface of the epidermis and papillary surface of the dermis. Collected keratinocytes were immersed in MCDB 153 (Sigma) with human keratinocyte growth supplements (bovine pituitary extract, $0.2 \% \mathrm{v} / \mathrm{v}$; bovine insulin, $5 \mu \mathrm{g} / \mathrm{mL}$; hydrocortisone, $0.18 \mu \mathrm{g} / \mathrm{mL}$; bovine transferrin, $5 \mu \mathrm{g} / \mathrm{mL}$, and human EGF, $0.2 \mathrm{ng} / \mathrm{mL}$ ) (Cascade Biologics, Portland, OR, U.S.A.) as culture medium in a $5 \% \mathrm{CO}_{2}$, $37^{\circ} \mathrm{C}$ incubator. The resulting cell suspension was then centrifuged at $150 \times g$ for $5 \mathrm{~min}$, and the cells were re-suspended in culture medium. A cell count was carried out using a hemocytometer, and trypan blue exclusion was used to assess cell viability. Keratinocytes were grown to approximately $60 \%$ confluence and passage in MCDB 153 culture 


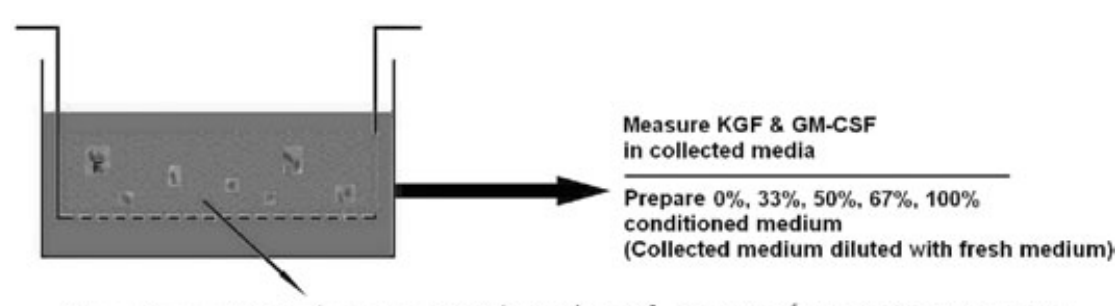

The collagen gel $\left(0.5 \mathrm{~cm}^{3}\right.$ contains $0,2 \times 10^{4}, 6 \times 10^{4}, 1 \times 10^{5}$, and $1.4 \times 10^{5}$ MMC-treated fibroblasts)

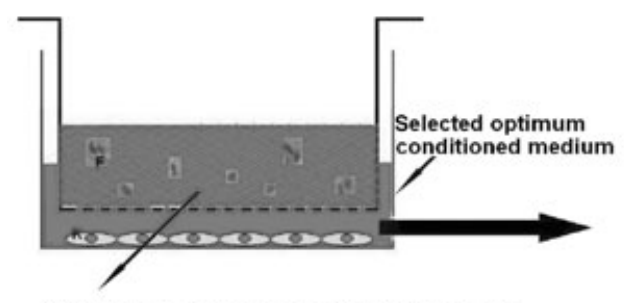

Passage by passage

Viability assay

Measure KGF \& GM-CSF

in collected media
FIG. 1. Schematic diagram of experimental design. Medium was collected every 3 days from MMC-treated fibroblasts. The collected media were diluted with fresh medium and used for keratinocyte culture.

With or without selected optimum feeder gel

medium. Second-, third-, fifth-, and seventh-passage keratinocytes were plated in specific medium containing $0.03 \mathrm{mM}$ calcium.

\section{Fibroblast isolation and culture}

Sections of dermis after keratinocyte isolation were washed several times in sterile phosphate buffered saline (PBS) and then finely minced with a scalpel. The minced dermis was incubated at $37^{\circ} \mathrm{C}$ overnight in $10 \mathrm{~mL}$ of a $0.5 \%$ collagenase A (Roche, Mannheim, Germany) solution. The collagenase digest was then centrifuged at $150 \times g$ for $10 \mathrm{~min}$, and the pellet was re-suspended in MCDB 105 containing 10\% fetal calf serum (FCS), $2 \mathrm{mM}$ glutamine, $0.625 \mathrm{mg} / \mathrm{mL}$ amphotericin B, $100 \mathrm{IU} / \mathrm{mL}$ penicillin, and $100 \mathrm{mg} / \mathrm{mL}$ streptomycin. Primary fibroblast isolation was produced and seeded into $15 \mathrm{~mL}$ MCDB 105 containing 10\% FCS. The cells were passaged by normal procedure once confluent. Fibroblasts at fifth passage were used in the experiment.

\section{MMC treatment}

The fifth passage subconfluent fibroblasts were treated with $10 \mu \mathrm{g} / \mathrm{mL}$ of MMC (Sigma) in 10\% FCS MCDB105 growth medium for $3 \mathrm{~h}$, rinsed with PBS, trypsinized, and re-suspended in prewarmed growth media for later use.

\section{Preparation of collagen feeder gel}

Collagen type 1 was extracted from rat tails by solubilization in $0.1 \%$ acetic acid. After centrifugation, the supernatant was collected and lyophilized at $-70^{\circ} \mathrm{C}$. Forty $\mathrm{mg}$ extracted collagen were dissolved in $10 \mathrm{~mL} 0.1 \%$ acetic acid and then mixed with $1.25 \mathrm{~mL}$ 10x MCDB 105 medium, which was neutralized by
$1 \mathrm{~N} \mathrm{NaOH}$. The solution of $0.4 \mathrm{~mL}$ was mixed with $0.1 \mathrm{~mL}$ of medium containing the appropriate number $\left(0,2 \times 10^{4}, 6 \times 10^{4}, 1 \times 10^{5}\right.$, and $\left.1.4 \times 10^{5}\right)$ of MMC-treated fibroblasts that was poured into transwell (BD Falcon, Franklin Lakes, NJ, U.S.A.) of 24well plates and allowed to form collagen feeder gel at $37^{\circ} \mathrm{C}$.

\section{Preparation of conditioned medium}

The gels with various fibroblast densities were fed with MCDB 105 containing 1\% FCS. The medium was collected and renewed every 3 days until 42 days. The collected media were filtered through a $0.22-\mu \mathrm{m}$ filter (Millipore, Billerica, MA, U.S.A.) and stored at $-20^{\circ} \mathrm{C}$. The interested growth factors in the collected medium are analyzed by enzyme-linked immunosorbent assay (ELISA). The collected medium is diluted with different ratio of culture medium as the conditioned medium for keratinocyte culture.

\section{KGF and GM-CSF measurement in collected medium: Determination of optimum collected medium}

Keratinocyte growth factor in the collected medium derived from variant fibroblast density was measured by ELISA. ELISA 96-well plates (Fisher Scientific, Agawan, MA, U.S.A.) were coated with $100 \mu \mathrm{L}$ of $1.0 \mu \mathrm{g} / \mathrm{mL}$ mouse antihuman KGF monoclonal antibody (R \& D Systems, Minneapolis, MN, U.S.A.) in PBS $/ 0.1 \%$ bovine serum albumin (BSA) for overnight incubation at room temperature. The antibody was removed the next day. The plate was washed three times with buffer solution (PBS/0.05\% Tween 20, pH 7.4) and blocked with $300 \mu \mathrm{L}$ of PBS 
with $1 \%$ BSA, $5 \%$ sucrose, and $0.05 \% \mathrm{NaN}_{3}$ for $1 \mathrm{~h}$ at room temperature. The plate was further washed three times with buffer solution and the collected medium was added in triplicate $(100 \mu \mathrm{L}$ per well) and incubated for $2 \mathrm{~h}$ at room temperature. The plate was washed three times and $100 \mu \mathrm{L}$ of biotinylated goat polyclonal antiKGF detection antibody ( $\mathrm{R} \& \mathrm{D}$ Systems) was added at $200 \mathrm{ng} / \mathrm{mL}$ in diluent (0.1\% BSA, $0.05 \%$ Tween 20 in Tris-buffered saline, $\mathrm{pH} 7.3$ ) for $2 \mathrm{~h}$ at room temperature. After three washes, $100 \mu \mathrm{L}$ of a horseradish peroxidase (HRP)conjugated avidin (Zymed Laboratories, South San Francisco, CA, U.S.A.) was added at a dilution of $1: 5000$ in $\mathrm{PBS} / 0.1 \% \mathrm{BSA}$ for $1 \mathrm{~h}$ at room temperature. The substrate $(10 \mathrm{mg} \quad o$-phenylenediamine dihydrochloride) and $10 \mu \mathrm{L}$ of $\mathrm{H}_{2} \mathrm{O}_{2}$ in $25 \mathrm{~mL}$ of substrate buffer $(5.1 \mathrm{mg} / \mathrm{mL}$ citric acid monohydrate and $13.78 \mathrm{mg} / \mathrm{mL} \mathrm{Na}_{2} \mathrm{HPO}_{4} \cdot 7 \mathrm{H}_{2} \mathrm{O}$ in $\mathrm{dH}_{2} \mathrm{O}$ ) were added $(100 \mu \mathrm{L}$ per well $)$ and the reaction was allowed to proceed for $10 \mathrm{~min}$ before addition of $50 \mu \mathrm{L}$ per well of $8 \mathrm{~N} \mathrm{H}_{2} \mathrm{SO}_{4}$ (stop solution). The optical density was read at $570 \mathrm{~nm}$ with an ELISA plate reader (ThermoMax plate reader, Molecular Devices, Palo Alto, CA, U.S.A.). GM-CSF in the collected media was also analyzed by the same technique according to the instructions of the manufacturer ( $\mathrm{R} \& \mathrm{D}$ Systems). The data were expressed in $\mathrm{pg} / \mathrm{mL}$. Each data represented the average of six repeats.

The collected medium with the highest concentration of KGF and GM-CSF is the optimum collected medium which will be diluted as the conditioned medium for keratinocyte proliferation test.

\section{Test of keratinocyte proliferation rate in optimum collected medium: Determination of optimum collagen feeder gel and optimum conditioned medium}

The optimum collected medium was diluted by fresh MCDB 153 medium with the ratio of $0: 1$, $1: 2,1: 1,2: 1$, and $1: 0$ (e.g., $0 \%, 33 \%, 50 \%, 67 \%$, and $100 \%$ diluted conditioned medium). The second-passage keratinocytes $\left(2 \times 10^{4}\right.$ cells/well $)$ were plated on 24-well plates. Cells were cultured in diluted conditioned medium $(0 \%, 33 \%, 50 \%$, $67 \%$, and $100 \%$ ) and collagen feeder gel was placed in each culture well. The collagen feeder gels with different MMC-treated fibroblasts were prepared at the following densities: cell-free, $2 \times 10^{4}, 6 \times 10^{4}, 1 \times 10^{5}$, and $1.4 \times 10^{5}$. After being cultured for 6 h, 3 days, 6 days, and 12 days, respectively, 3-(4,5-dimethylthiazol-2-yl)-2,5-diphenyl-2Htetrazolium bromide (MTT) (Sigma) was added to each well and further cultured for $3 \mathrm{~h}$ at $37^{\circ} \mathrm{C}$. The dark blue crystals were lysed with acidified isopropanol (2 $\mathrm{mM} \mathrm{HCl}$ in isopropanol) and the absorbance at $570 \mathrm{~nm}$ was measured. The data was averaged by six repeated experiments.

\section{Cell viability evaluation: Study of the importance of optimum collagen feeder gel and optimum conditioned medium}

Previously, optimum conditioned medium and optimum collagen feeder gel were determined. This step studies the importance of these two factors for the keratinocyte proliferation. The experiment procedures are briefly described as follows.

The third-, fifth-, and seventh-passage keratinocytes were seeded with the density of $2 \times 10^{4}$ cells/well in a 24-well tissue-culture tray either in fresh MCDB 153 medium without supplements ( $0 \%$ conditioned medium) or in optimum conditioned medium. Keratinocytes were cocultured with optimum feeder gel or without optimum feeder for 6 days. MTT assay was used to measure the keratinocyte viability. The culture medium was renewed every 3 days and collected for growth factor analysis. Each value represented the average of six wells.

\section{Immunoassay of keratin 10 (K10)}

Immunoassay of K10 could indicate the keratinocyte differentiation. The keratinocyte on glass slides were washed twice with ice-cold PBS and fixed in acetone/methanol $1: 1$ for $20 \mathrm{~min}$ at $-20^{\circ} \mathrm{C}$. Endogenous peroxidase activity was blocked with $3 \% \quad \mathrm{H}_{2} \mathrm{O}_{2}$ at room temperature. After blocking unspecific binding sites with $3 \%$ BSA in PBS, slides were incubated overnight at $4{ }^{\circ} \mathrm{C}$ with antibodies against $\mathrm{K} 10$ ( $1: 400$ dilution in blocking solution; $30 \mathrm{~min}$ at room temperature) (Chemicon International, Temecula, CA, U.S.A.). Slides were then washed five times with PBS and incubated with $50 \mu \mathrm{L}$ horse antimouse biotinylated antibody $(1: 200$ in blocking solutions) for $30 \mathrm{~min}$ at room temperature. The slides were then incubated with avidinHRP for $30 \mathrm{~min}$ and developed with a substrate kit (Vector Laboratories, Burlingame, CA, U.S.A.) according to manufacturer's recommendations. Slides were washed five times with PBS, followed by a 5 min wash with tap $\mathrm{H}_{2} \mathrm{O}$ and counterstained with hematoxylin (15 s with Richard-Allan hematoxylin or $50 \mathrm{~s}$ with Harris hematoxylin). The slides were washed with tap $\mathrm{H}_{2} \mathrm{O}$ for $10 \mathrm{~min}$ and mounted with an aqueous mounting medium (Crystal/Mount, Biomeda, Foster City, CA, U.S.A.). Using standardized imaging analysis, positive surface area of K10 was measured. 


\section{TUNEL test}

To detect apoptotic keratinocyte death, cells were stained using an "In Situ Cell Death Detection Kit, POD" (Roche). It is based on the TUNEL method. Briefly, cells were fixed for $1 \mathrm{~h}$ in $4 \%$ paraformaldehyde in PBS at room temperature. Endogenous peroxidase was inactivated by incubation with $2 \% \mathrm{H}_{2} \mathrm{O}_{2}$ dissolved in methanol for $10 \mathrm{~min}$ at room temperature. Cells were permeabilized with $0.1 \%$ Triton X100 for $2 \mathrm{~min}$ on ice. After that, cells were labeled by incubation with TUNEL reaction mixture at $37^{\circ} \mathrm{C}$ for $1 \mathrm{~h}$. The cells were washed three times with PBS and then incubated with peroxidase-converter at $37^{\circ} \mathrm{C}$ for $30 \mathrm{~min}$. Then the cells were washed and stained with fresh $0.05 \%$ diaminobenzidine. Using standardized image analysis, the positive surface area of TUNEL stain was measured.

\section{Population doubling time}

To determine population doubling time, single keratinocyte cells were cultured in 24-well culture plates using $0 \%$ or $67 \%$ conditioned media. The cells were grown for 7 days with feeder gel or without feeder gel. The plates were stained using $0.2 \%$ crystal violet in $10 \%$ ethanol for $10 \mathrm{~min}$, and cells were counted.

\section{Data analysis}

Results were expressed as the mean $\pm \mathrm{SD}$. Oneway ANOVA was used for comparisons of experimental groups to a control group, as well as for pair wise comparisons among experimental groups. $P<0.05$ was considered statistically significant.

\section{RESULTS}

The concentrations of KGF and GM-CSF in collected medium from various collagen feeder gels are shown in Fig. 2. The results revealed that the concentration of KGF (Fig. 2a) and GM-CSF (Fig. 2b) both increased with the cell density in the collagen feeder gel. The concentrations of KGF and GM-CSF did not increase when the cell density was greater $1 \times 10^{5}$ cells/gel. The MMC-treated fibroblasts embedded in the collagen gel constantly secreted steady amounts of KGF and GM-CSF even after 42-day culture. The concentrations of KGF in the collected medium secreted from collagen feeder gels with different cell density were in the range of $0 \mathrm{pg} / \mathrm{mL}$ (cell-free) to $110 \mathrm{pg} / \mathrm{mL}\left(1 \times 10^{5}\right.$ cells $/$ gel $)$. The concentrations of GM-CSF were in the range of $0 \mathrm{pg} / \mathrm{mL}$ (cell-free) to $60 \mathrm{pg} / \mathrm{mL}\left(1 \times 10^{5}\right.$ and $1.4 \times 10^{5}$ cells $/$ gel $)$. From this study, collagen feeder gel with cell density of $1 \times 10^{5}$
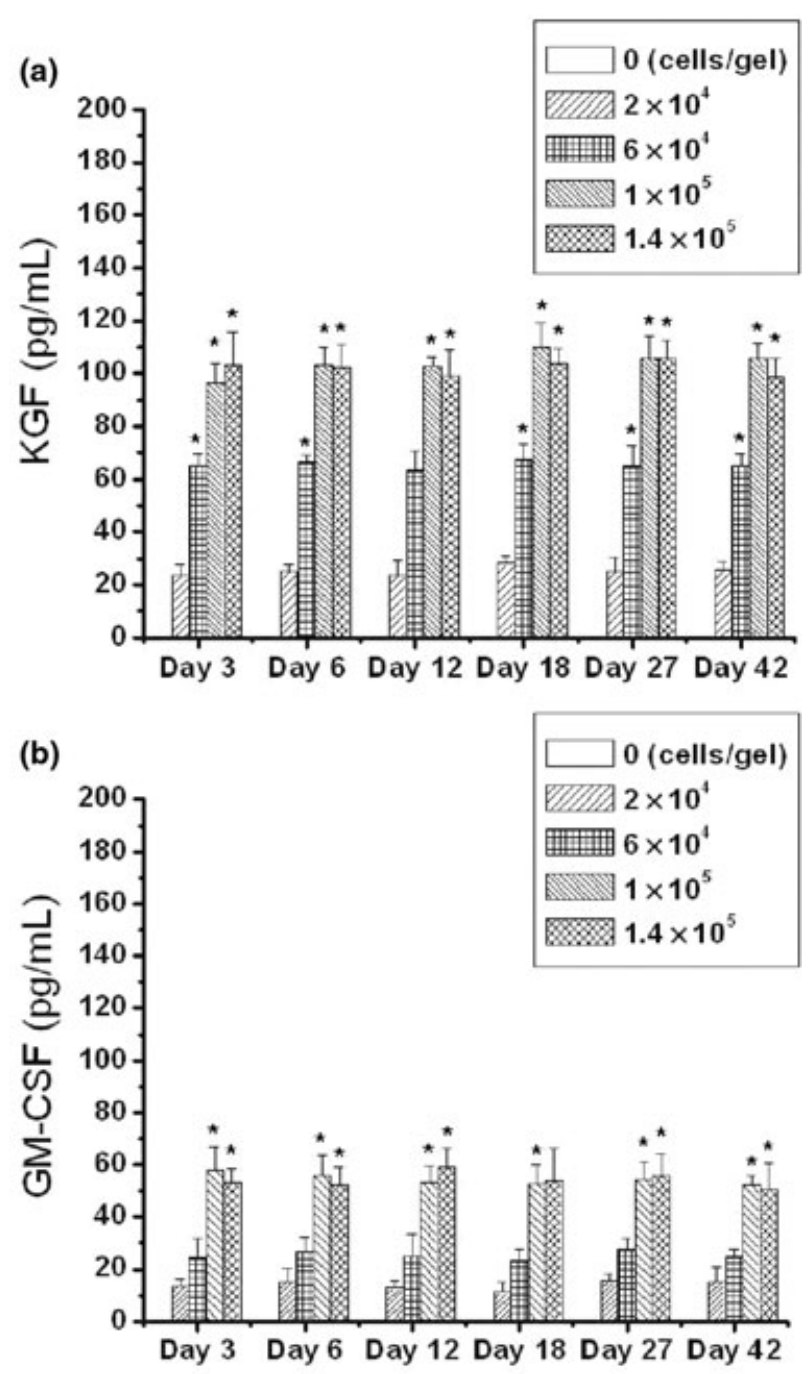

FIG. 2. Concentrations of (a) KGF and (b) GM-CSF in media collected at days $3,6,12,18,27$, and 42 . Error bars represent means with standard deviation. $n=6,{ }^{*} P<0.05$.

cells/gel was the optimum cell density to produce optimum collected medium.

The optimum collected medium was diluted with MCBD 153 cultured medium in different ratios as conditioned medium, which were used to culture keratinocytes with different collagen feeder gels. Figure 3 shows the keratinocyte viability cocultured with different cell densities of collagen feeder gels in different conditioned mediums. After being seeded for $6 \mathrm{~h}$, keratinocytes cultured in $67 \%$ conditioned medium showed the highest viability at all cell densities of collagen of feeder gels (Fig. 3a). After being cultured in conditioned medium and cocultured with collagen feeder gel for 3 days (Fig. 3b), 6 days (Fig. 3c), and 12 days (Fig. 3d), the keratinocytes appeared to have higher proliferation rate in the 
(a)

$6 \mathrm{~h}$

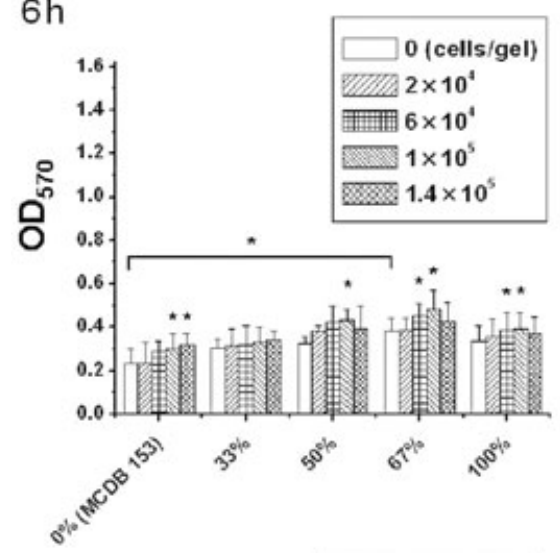

(c)

$6 \mathrm{~d}$

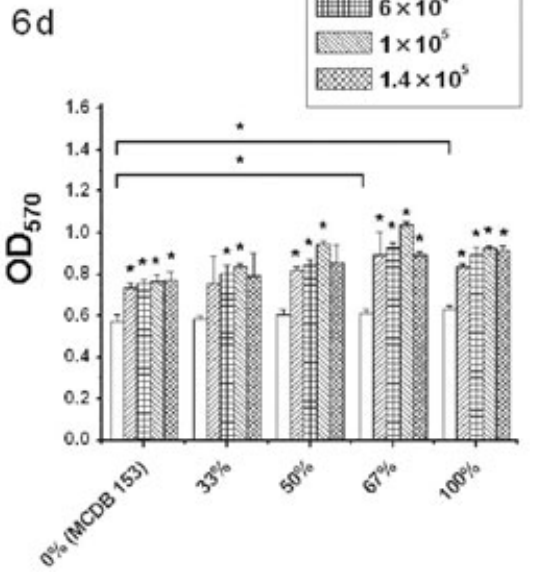

(b)

$3 d$

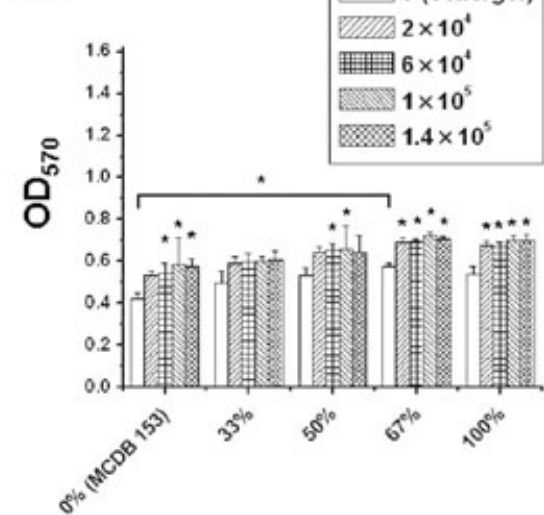

(d)

$12 \mathrm{~d}$

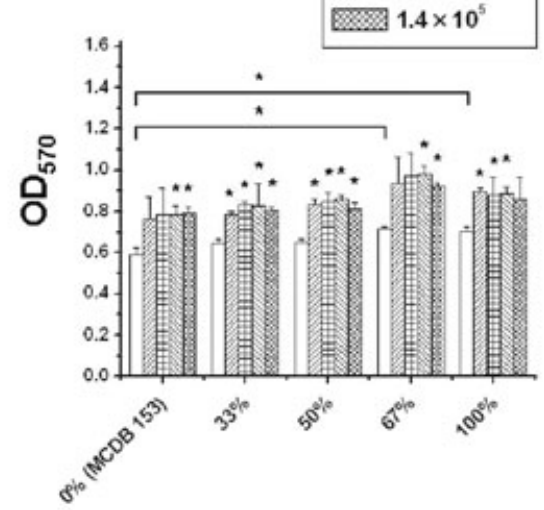

FIG. 3. MTT assay of keratinocytes cultured with collagen feeder gel at different cell densities in different conditioned media. (a) $6 \mathrm{~h}$, (b) 3 days, (c) 6 days, and (d) 12 days. Error bars represent means with standard deviation. $n=6,{ }^{*} P<0.05$.
$67 \%$ conditioned medium. As shown in Fig. 3, the keratinocytes cocultured with $1 \times 10^{5}$ cells/gel density of feeder gel had better proliferation rate. The results concluded that $67 \%$ conditioned medium and $1 \times 10^{5}$ cells/gel density feeder gel were the optimum conditioned medium and optimum cell density in feeder gel for keratinocyte proliferation, respectively.

The phase contrast photographs of the keratinocytes cocultured with feeder gel $\left(1 \times 10^{5}\right.$ cells/gel $)$ in $67 \%$ conditioned medium are shown in Fig. 4. Pic- tures showed that the keratinocytes cultured for 3 days, 6 days, and 12 days were in preconfluence (Fig. 4a), confluence (Fig. 4b), and postconfluence (Fig. 4c), respectively. The keratinocytes in preconfluent state had extended cell body and stretched out filaments to explore surroundings (Fig. 4a). In contrast, keratinocytes exhibited cubical shape in the confluent state (Fig. 4b) and stratified shape in the postconfluent state (Fig. 4c).

Immunoassay of the K10 was used to check if keratinocytes in confluent state would have gone
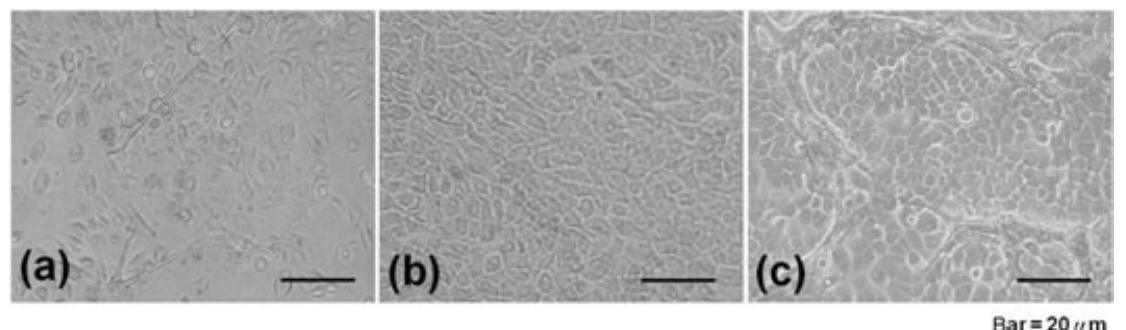

FIG. 4. Phase contrast photographs of keratinocytes with optimum feeder gel in $67 \%$ conditioned medium at the condition of (a) preconfluence, (b) confluence, and (c) postconfluence. The duplicate measurements were performed in three independent experiments. 


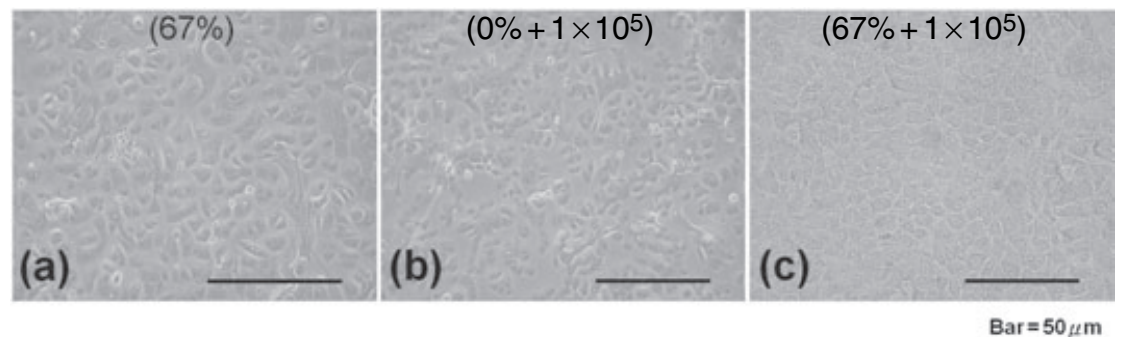

FIG. 5. Immunoassay of keratin 10 of keratinocytes (confluence). Keratinocytes cultured (a) in $67 \%$ conditioned medium $(67 \%)$, (b) with optimum feeder gel $\left(1 \times 10^{5}\right.$ cells/gel) in MCDB 153 medium $\left(0 \%+1 \times 10^{5}\right)$, and (c) with optimum feeder gel in $67 \%$ conditioned medium $\left(67 \%+1 \times 10^{5}\right)$. All samples are keratin 10-negative. The duplicate measurements were performed in three independent experiments. toward undesired differentiation. Figure 5a shows the keratinocytes cultured in $67 \%$ conditioned medium without feeder gel for 6 days (confluence). Figure $5 \mathrm{~b}$ shows the keratinocytes cocultured with feeder gel $\left(1 \times 10^{5}\right.$ cells/gel $)$ in MCDB 153 medium $(0 \%$ conditioned medium $)$ for 6 days. Figure $5 \mathrm{c}$ shows the keratinocytes cocultured with feeder gel $\left(1 \times 10^{5}\right.$ cells/gel $)$ in $67 \%$ conditioned medium for 6 days. The three experimental groups all showed negative in K10 expression. The results concluded that the keratinocytes have not gone differentiation under the confluent condition.

Figure 6 is the immunoassay of K10 of keratinocytes in postconfluent state. Figure 6a is keratinocytes cultured in $67 \%$ conditioned medium without feeder gel for 12 days (postconfluence). Figure $6 \mathrm{~b}$ is the keratinocytes cocultured with feeder gel $\left(1 \times 10^{5}\right.$ cells/gel $)$ in MCDB 153 medium for 12 days. Figure $6 \mathrm{c}$ is the keratinocytes cocultured with feeder gel $\left(1 \times 10^{5}\right.$ cells/gel $)$ in $67 \%$ conditioned medium for 12 days. The three experimental groups all showed positive and different levels of K10. The keratinocytes cultured in $67 \%$ conditioned medium without feeder gel showed the highest expression in
K10 and the keratinocytes cocultured with feeder gel $\left(1 \times 10^{5}\right.$ cells/gel $)$ in $67 \%$ conditioned medium had the lowest value in K10 expression (Fig. 6d).

Keratinocytes in postconfluent state (overcrowd) would have gone toward undesired differentiation. If keratinocytes are cultured under optimum condition (cocultured with feeder gel $\left[1 \times 10^{5}\right.$ cells/gel] in $67 \%$ conditioned medium), it inhibits or delays the differentiation. Furthermore, the keratinocytes cocultured with $1 \times 10^{5}$ cells/gel feeder gel in MCDB 153 medium had lower K10 expression than those cultured in $67 \%$ conditioned medium without feeder gel. The optimum feeder gel effectively inhibited keratinocyte differentiation over optimum condition medium.

TUNEL stain is utilized to check DNA fragmentation that normally associates with cell apoptosis. Figure $7 \mathrm{a}$ shows the highest apoptosis rate while Fig. $7 \mathrm{c}$ shows the lowest. Feeder gel $\left(1 \times 10^{5}\right.$ cells/gel $)$ in $67 \%$ conditioned medium provided the best cultured condition to prevent apoptosis. In conclusion, the optimum feeder gel is more important than the optimum conditioned medium in both keratinocyte proliferation and inhibition of apoptosis.
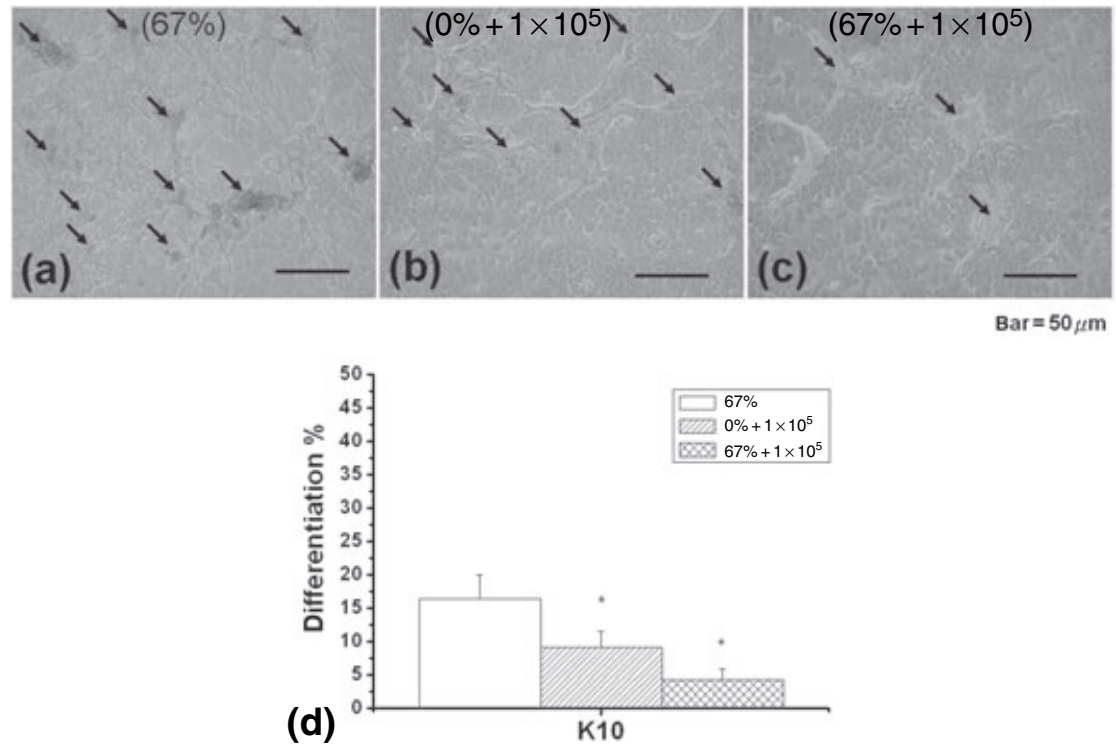

FIG. 6. Immunoassay of keratin 10 of keratinocytes (postconfluence). Keratinocytes cultured (a) in $67 \%$ conditioned medium $(67 \%)$, (b) with optimum feeder gel $\left(1 \times 10^{5}\right.$ cells/gel $)$ in MCDB 153 medium $\left(0 \%+1 \times 10^{5}\right)$, and (c) with optimum feeder gel in $67 \%$ conditioned medium $\left(67 \%+1 \times 10^{5}\right)$. (d) The percentage of keratin 10-positive area in total area. Error bars represent means with standard deviation. $n=6,{ }^{*} P<0.05$. 

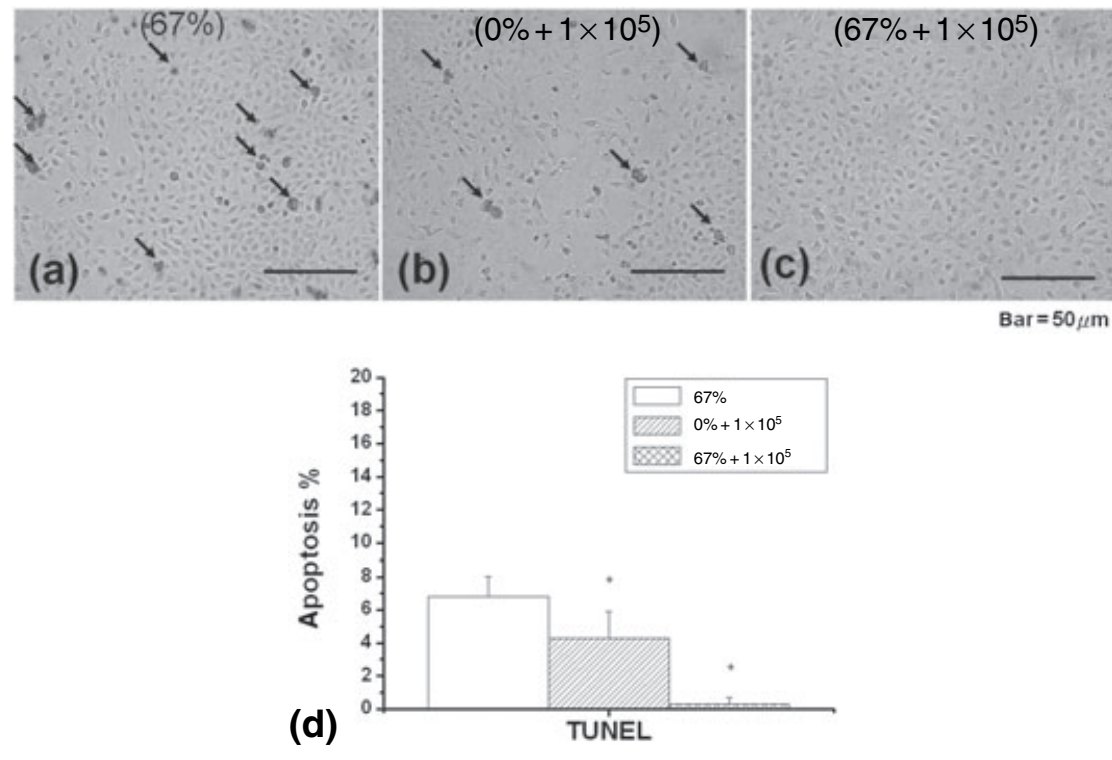

FIG. 7. TUNEL assay of keratinocytes (confluence). Keratinocytes cultured (a) in $67 \%$ conditioned medium (67\%), (b) with optimum feeder gel $\left(1 \times 10^{5}\right.$ cells/gel $)$ in MCDB 153 medium $\left(0 \%+1 \times 10^{5}\right)$, and (c) with optimum feeder gel in $67 \%$ conditioned medium $\left(67 \%+1 \times 10^{5}\right)$. (d) The percentage of apoptotic area in total area. Error bars represent means with standard deviation. $n=6,{ }^{\star} P<0.05$.
The next set of experiments checked the keratinocyte viability at different passages (third-, fifth-, and seventh-passage keratinocytes) with different conditions, from which the optimum cultured condition will be determined. As shown in Fig. 8, the via-

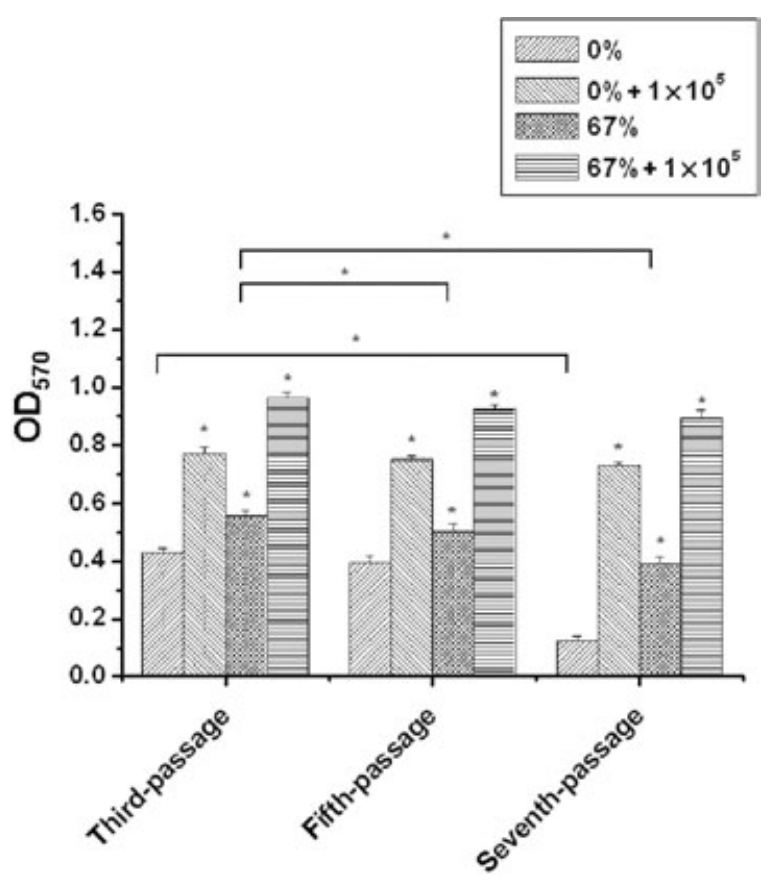

FIG. 8. Viability of third-, fifth-, and seventh-passage keratinocytes cultured in MCDB 153 medium (0\%), or cultured with optimum feeder gel in MCDB 153 medium $\left(0 \%+1 \times 10^{5}\right)$, or cultured in $67 \%$ conditioned medium $(67 \%)$, or cultured with optimum feeder gel in $67 \%$ conditioned medium $\left(67 \%+1 \times 10^{5}\right)$ for 6 days. Error bars represent means with standard deviation. $n=6,{ }^{*} P<0.05$. bility of keratinocytes cultured in MCDB 153 medium significantly decreased with the number of passages. When keratinocytes cocultured with $1 \times 10^{5}$ cells/gel in MCDB 153 medium, the viabilities did not change much for all different passages, but were much higher than those that were not cocultured in MCDB 153 medium. The keratinocyte viability gradually decreased as number passages increased when cultured in $67 \%$ conditioned medium. The viability showed no significant decrease when the keratinocytes were cocultured with $1 \times 10^{5}$ cells/gel feeder gel in $67 \%$ conditioned medium. In conclusion, the keratinocytes cocultured with $1 \times 10^{5}$ cells/gel feeder gel in $67 \%$ conditioned medium had the highest viability and retained their viability as passage increased. The optimum feeder gel played a more important factor in keratinocyte viability than that of the optimum conditioned medium.

Keratinocytes cultured in 0 and $67 \%$ conditioned medium without feeder gel showed similar population doubling numbers over 6 days of $2.61 \pm 0.3$ and $2.89 \pm 0.4$, respectively. Nevertheless, keratinocytes cultured in 0 and $67 \%$ conditioned medium with feeder gel had doubling numbers of $4.35 \pm 0.3$ and $4.76 \pm 0.2$, respectively.

In Fig. 9a, keratinocytes cultured in MCDB 153 did not secrete KGF. If keratinocytes were cultured with optimum feeder gel (with $1 \times 10^{5}$ cells/gel) in MCDB 153 medium, the concentration of KGF increased to $250 \mathrm{pg} / \mathrm{mL}$ in the medium. Keratinocytes cultured in $67 \%$ conditioned medium showed that KGF level in the medium was only about $70 \mathrm{pg} / \mathrm{mL}$. Keratinocytes cultured with optimum feeder gel (with $1 \times 10^{5}$ cells/ gel) in $67 \%$ conditioned medium showed that the 

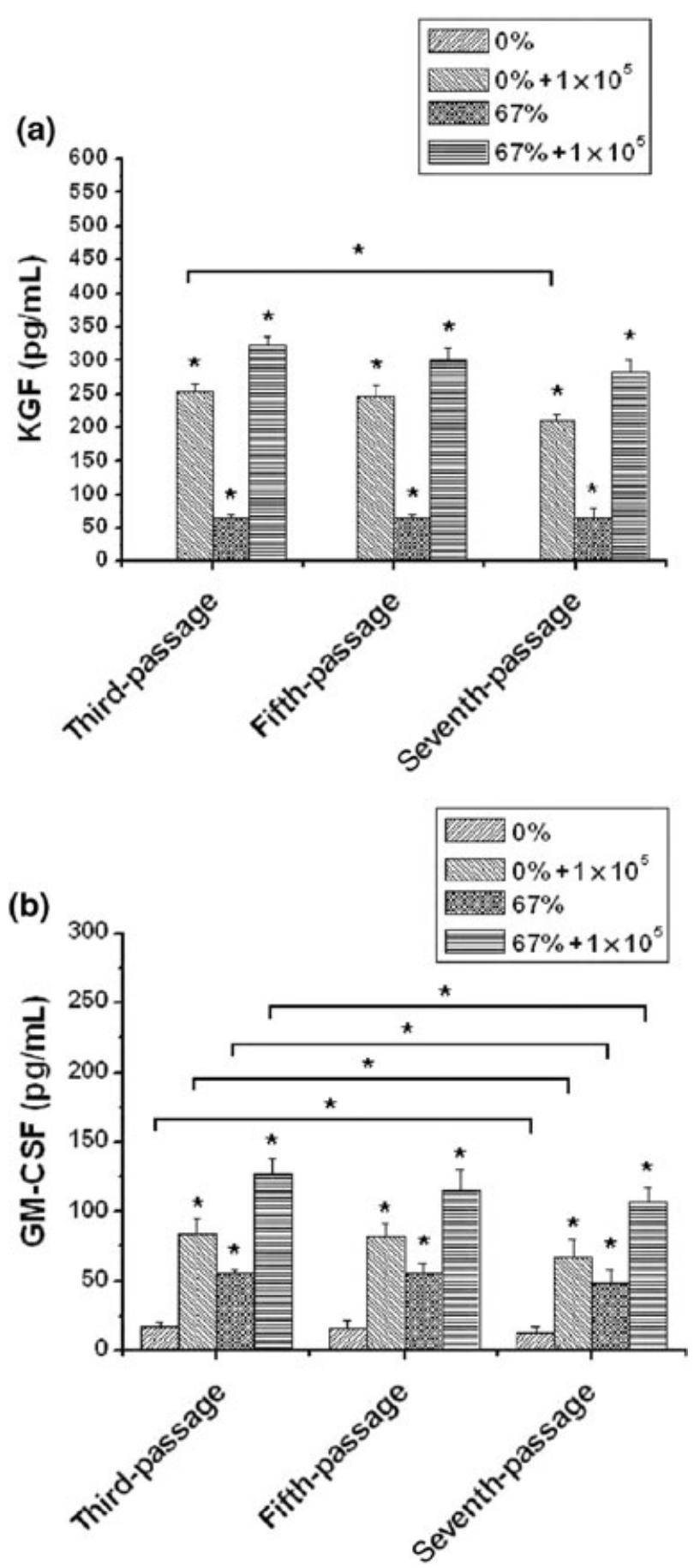

FIG. 9. Concentrations of (a) KGF and (b) GM-CSF in media collected at day 3. Keratinocytes cultured in MCDB 153 medium $(0 \%)$, or cultured with optimum feeder gel in MCDB 153 medium $\left(0 \%+1 \times 10^{5}\right)$, or cultured in $67 \%$ conditioned medium $(67 \%)$, or cultured with optimum feeder gel in $67 \%$ conditioned medium $\left(67 \%+1 \times 10^{5}\right)$. Error bars represent means with standard deviation. $n=6,{ }^{*} P<0.05$. concentration of KGF was highest at around $300 \mathrm{pg} /$ $\mathrm{mL}$ in the medium. In Fig. 9b, keratinocytes cultured in MCDB 153 secreted $15 \mathrm{pg} / \mathrm{mL}$ GM-CSF in the medium. When keratinocytes were cultured with optimum feeder gel with $1 \times 10^{5}$ cells/gel in MCDB 153 medium, the concentration of GM-CSF was about $82 \mathrm{pg} / \mathrm{mL}, 80 \mathrm{pg} / \mathrm{mL}$, and $72 \mathrm{pg} / \mathrm{mL}$ for the third, fifth, and seventh passage, respectively. Keratinocytes cultured in $67 \%$ conditioned medium secreted GM-CSF at roughly $50 \mathrm{pg} / \mathrm{mL}$ in the medium. If keratinocytes cultured with feeder gel in $67 \%$ conditioned medium, the concentration of GM-CSF was about $128 \mathrm{pg} / \mathrm{mL}$, $119 \mathrm{pg} / \mathrm{mL}$, and $110 \mathrm{pg} / \mathrm{mL}$ for the third, fifth, and seventh passage, respectively. In conclusion, the level of $\mathrm{KGF}$ in the medium shows no significant differences with the passages but the GM-CSF level in the medium slightly decreases with the passages.

\section{DISCUSSION}

At the lower cell density in the gel, the number of fibroblasts are insufficient to supply adequate KGF and GM-CSF to the environment. When cell density in the feeder gel was greater than $1 \times 10^{5}$ cells/gel, KGF and GM-CSF levels in the medium did not increase due to high concentration of growth factor in the medium causing inhibition feedback $(14,15)$.

From the results of Fig. 3, 67\% conditioned medium was the optimum medium for keratinocyte proliferation. Previous studies showed that KGF triggers PI3K/Akt response and induces cell protection through modulation of intermediate proteins located upstream from terminal caspases. KGF can preserve epithelial cell integrity by inhibiting apoptosis. The protection is mediated in part through activation of the PI3K survival pathway (16). In addition, stimulation of keratinocyte proliferation can be directly brought about by GM-CSF itself (17), and invoked by the GM-CSF-mediated release of other cytokines and growth factors (18-20). Thus, KGF and GM-CSF could promote keratinocyte proliferation (Fig. 3) and inhibit keratinocyte apoptosis (Fig. 7). If keratinocytes were cultured in lower ratios of conditioned medium, KGF and GM-CSF were insufficiently supplied to the culture system. Keratinocytes cultured in $100 \%$ conditioned medium did not proliferate properly. This is because $100 \%$ conditioned medium contains more metabolic wastes than other conditioned media. In Fig. 3, the feeder gel with a cell density of $1 \times 10^{5}$ cells/gel is the optimum cell density for coculturing with keratinocyte proliferation. This is because lower cell densities in the feeder gel are insufficient in providing growth factor and higher cell 
density did not effectively increase the amount of growth factor.

Keratin 10 is the early stage differentiation marker of keratinocytes $(21,22)$. In the study, keratin 10 did not express in the confluence condition (Fig. 5) but appears in the postconfluent condition (Fig. 6). Based on the results of Figs. 5 and 6, keratinocytes did not proliferate properly under postconfluent condition.

As shown in Fig. 7, keratinocytes cultured with optimum feeder gel performed better than keratinocytes cultured in $67 \%$ conditioned medium in the prevention of apoptosis. Fibroblasts in the feeder gel provided real-time communication with the keratinocytes and secreted necessary growth factors for keratinocytes. Furthermore, cell apoptosis was significantly delayed if the keratinocytes were cultured with optimum feeder gel in $67 \%$ conditioned medium (Fig. 7c). Results of Figs. 8 and 9 showed that keratinocytes cultured with optimum feeder gel in $67 \%$ conditioned medium kept their viability and continued to secrete necessary growth factors until the seventh passage.

\section{CONCLUSION}

From the study of apoptosis, the keratinocytes should not be cultured in postconfluent conditions due to undesired differentiation. From the study of cell viability per passage, the optimum feeder gel is more important to the keratinocyte proliferation compared with optimum condition medium. Optimum feeder gel in $67 \%$ conditioned medium effectively promoted proliferation, inhibited apoptosis, and prevented keratinocyte from differentiation. This combination of conditioned media and feeder gel to culture keratinocytes minimize the usage of expensive external supplements. The results concluded that keratinocyte cultivation in feeder gel with modified medium should be feasible in the production of high quality keratinocytes for skin equivalents preparation.

Acknowledgments: The medical ethical committee of the National Taiwan University Hospital approved all studies described. The authors thank L.T. Chen, Y.L. Lee, and T.M. Chen for critical and helpful comments during the preparation of this manuscript. This work was supported by a stipend from the National Science Council.

\section{REFERENCES}

1. Brigham PA, McLoughlin E. Burn incidence and medical care use in the United States: estimates, trends, and data sources. J Burn Care Rehabil 1996;17:95-107.
2. Potten CS, Morris RJ. Epithelial stem cells in vivo. J Cell Sci 1988;10(Suppl.):45-62.

3. Kato Y, Tapping RI, Huang S, Watson MH, Ulevitch RJ, Lee JD. Bmk1/Erk5 is required for cell proliferation induced by epidermal growth factor. Nature 1998;15:713-6.

4. Rheinwald JG, Green H. Epidermal growth factor and the multiplication of cultured human epidermal keratinocyte. Nature 1977;265:421-4.

5. Gibbs S, Silva Pinto AN, Murli S, Huber M, Hohl D, Ponec M. Epidermal growth factor and keratinocyte growth factor differentially regulate epidermal migration, growth, and differentiation. Wound Repair Regen 2000;8:192-203.

6. El-Ghalbzouri A, Gibbs S, Lamme E, Van Blitterswijk CA, Ponec M. Effect of fibroblasts on epidermal regeneration. $\mathrm{Br}$ J Dermatol 2002;147:230-43.

7. Brauchle M, Angermeyer K, Hubner G, Werner S. Large induction of keratinocyte factor expression by serum growth factors and pro-inflammatory cytokines in cultured fibroblasts. Oncogene 1994;9:3199-204.

8. Cook PW, Pittelkow MR, Shipley GD. Growth factorindependent proliferation of normal human neonatal keratinocyte: production of autocrine- and paracrine-acting mitogenic factors. J Cell Physiol 1991;146:277-89.

9. Finch PW, Rubin JS, Miki T, Ron D, Aaronson SA. Human KGF is FGF-related with properties of a paracrine effector of epithelial cell growth. Science 1989;245:752-5.

10. Parshley MS, Simm HS. Cultivation of adult skin epithelial cells (chicken and human) in vitro. Am J Anat 1950;86:163-9.

11. Coulomb B, Dubertret L, Merrill C, Touraine R, Bell E. The collagen lattice: a model for studying epidermalization in vitro. Br J Dermatol 1984;114:91-101.

12. Smola H, Thiekötter G, Fusenig NE. Mutual induction of growth factor gene expression by epidermal-dermal cell interaction. J Cell Biol 1993;122:417-29.

13. Occleston NL, Alexander RA, Mazure A, Larkin G, Khaw PT. Effects of single exposures to antiproliferative agents on ocular fibroblast-mediated collagen contraction. Invest Ophthalmol Vis Sci 1994;35:3681-90.

14. Yamasaki K, Toriu N, Hanakawa Y, et al. Keratinocyte growth inhibition by high-dose epidermal growth factor is mediated by transforming growth factor beta autoinduction: a negative feedback mechanism for keratinocyte growth. J Invest Dermatol 2003;120:1030-7.

15. Umbarger HE. Evidence for a negative-feedback mechanism in the biosynthesis of isoleucine. Science 1956;123:848.

16. Bao S, Wang Y, Sweeney P, et al. Keratinocyte growth factor induces Akt kinase activity and inhibits Fas-mediated apoptosis in A549 lung epithelial cells. Am J Physiol Lung Cell Mol Physiol 2005;288:L36-42.

17. Breuhahn K, Mann A, MuÈller K, et al. Overexpression of GM-CSF in the epidermis of transgenic mice induces both keratinocyte proliferation and apoptosis. Cell Growth Differentiation 2000;11:111-21.

18. Valente AJ, Xie JF, Abramova MA, Wenzel UO, Abboud HE, Graves DT. A complex element regulates IFN-gammastimulated monocyte chemoattractantprotein-1 gene transcription. J Immunol 1998;161:3719-28.

19. Gasperini S, Marchi M, Calzetti F. Gene expression and production of the monokine induced by IFN-gamma (MIG), IFN-inducible T cell alphachemoattractant (I-TAC), and IFNgamma-inducible protein-10 (IP-10) chemokines by human neutrophils. J Immunol 1999;162:4928-37.

20. Li Q, Normolle DP, Sayre DM. Immunological effects of BCG as an adjuvant in autologous tumor vaccines. Clin Immunol 2000;94:64-72.

21. Roop DR, Hawley-Nelson P, Cheng CK, Yuspa SH. Keratin gene expression in mouse epidermis and cultured epidermal cells. Proc Natl Acad Sci USA 1983;80:716-20.

22. Schweizer J, Kinjo M, Furstenberger G, Winter H. Sequential expression of mRNA-encoded keratin sets in neonatal mouse epidermis: basal cells with properties of terminally differentiating cells. Cell 1984;37:159-70. 